\title{
Prevalence of Aggressive Periodontitis in Patients visiting the OPD of Periodontology, RajaRajeswari Dental College \& Hospital, Bengaluru
}

\author{
${ }^{1}$ Shilpa Shivanand, ${ }^{2}$ Navnita Singh, ${ }^{3}$ Shivaprasad Bilichodmath
}

\begin{abstract}
Introduction: The prevalence of aggressive periodontitis (AP) has been extensively studied globally. Limited information is available on the AP prevalence in the Indian population. Hence, a survey was undertaken to give a more accurate prevalence of AP in the population visiting the outpatient Department of Periodontology, RajaRajeswari Dental College \& Hospital, Bengaluru, Karnataka, India.

Materials and methods: A total of 500 patients were screened to estimate the prevalence of AP. Thorough gingival examination, including bleeding on probing, was done to evaluate the gingival condition. The periodontal status was evaluated by assessment of clinical attachment level, probing pocket depth, and gingival recession. The subjects provisionally diagnosed with AP were referred to the Department of Radiology for further examination. The radiographic assessment involved full mouth intraoral periapical radiographs and panoramic radiographs.
\end{abstract}

Results: Out of the 500 patients screened, 8 cases (LAP-4, GAP-4) were found to be confirmed with the diagnosis of AP, giving a prevalence of $1.6 \%$.

Conclusion: The prevalence rate of AP in the screened population is $1.6 \%$. The higher prevalence of AP in the present study can be attributed to the fact that the population studied is hospital based because of the convenience. The prevalence of AP is highly variable and controversial globally and needs a concerted and systemic approach if this is to be settled.

Keywords: Aggressive periodontitis, Bengaluru, India, Prevalence.

How to cite this article: Shivanand S, Singh N, Bilichodmath $\mathrm{S}$. Prevalence of Aggressive Periodontitis in Patients visiting the OPD of Periodontology, RajaRajeswari Dental College \& Hospital, Bengaluru. J Health Sci Res 2015;6(2):37-40.

Source of support: Nil

Conflict of interest: None

\section{INTRODUCTION}

Aggressive periodontitis (AP) is characterized by the rapid rate of disease progression seen in an otherwise

\footnotetext{
${ }^{1,2}$ Postgraduate Student, ${ }^{3}$ Postgraduate Guide

${ }^{1-3}$ Department of Periodontology, RajaRajeswari Dental College \& Hospital, Bengaluru, Karnataka, India

Corresponding Author: Shilpa Shivanand, Postgraduate Student, Department of Periodontology, RajaRajeswari Dental College \& Hospital, Bengaluru, Karnataka, India, Phone: +918861785059, e-mail: shlpshivanand@gmail.com
}

healthy individual, absence of large accumulations of plaque and calculus, and a family history of aggressive disease suggestive of genetic inheritance. Aggressive periodontitis is further classified into a localized form and a generalized form. ${ }^{1}$ Black, in the year $1886,{ }^{2}$ used the terms phagedenic pericementitis and chronic suppurative pericementitis to describe patients who suffered from a rapid destruction of the alveolar bone. Gottlieb in the year 1923 described an unusual form of periodontal disease that involved some or all of the permanent incisors and first molars of young individuals.

\section{PREVALENCE}

Global prevalence of AP remains elusive. ${ }^{3}$ Estimates of AP vary widely from 0 to $0.17 \%$. The rate appears to be $0.1 \%$ in the developed nations and $5 \%$ in the underdeveloped nations. ${ }^{4}$ Lower prevalence rates ranging between 0.1 and $0.2 \%$ have been reported in Europe, ${ }^{5}$ whereas high prevalence rates have been reported in Brazil (3.8\%), ${ }^{6}$ Iraq $(11.5 \%),{ }^{7}$ Indonesia $(8 \%),{ }^{8}$ and the United States $(10 \%){ }^{9}$ There is sparse information about the prevalence of AP in India. A survey of 1000 subjects of the 12 to 16 year age group (500 males, 500 females) in schools and colleges of Hyderabad city reported a prevalence of periodontosis of $0.5 \%,{ }^{10}$ while in another study in the same city, prevalence was reported at $0.45 \% .{ }^{11}$ Reddy ${ }^{12}$ reported a prevalence of $0.3 \%$ of localized aggressive periodontitis (LAP) cases in the Khammam district of Andhra Pradesh.

Although attributed to methodological issues, diagnostic techniques, sample bias, and changing definition of what constitutes $\mathrm{AP}$, the wide variation in the prevalence of AP is difficult to fathom and has been a fertile area of research. Hence, the aim of the present study was to estimate the prevalence of AP in patients visiting the outpatient department (OPD) of Periodontology, RajaRajeswari Dental College \& Hospital, Bengaluru, Karnataka, India.

\section{MATERIALS AND METHODS}

The study protocol was approved by the Institutional Ethics Committee and consent from all participants was taken for clinical examination. Individual informed consent was obtained from all confirmed cases of AP for detailed clinical and radiographic assessment. 
Aggressive periodontitis was diagnosed according to the 1999 American Academy of Periodontology classification into either LAP or generalized aggressive periodontitis (GAP). ${ }^{13}$

\section{LOCALIZED AGGRESSIVE PERIODONTITIS}

Localized aggressive periodontitis usually has an age of onset at about puberty. Clinically, it is characterized as having localized first molar/incisor presentation with interproximal attachment loss on at least two permanent teeth, one of which is a first molar, and involving no more than two teeth other than first molars and incisors. Radiographic findings may include an arc-shaped loss of the alveolar bone extending from the distal surface of the second premolar to the mesial surface of the second molar. $^{13}$

\section{GENERALIZED AGGRESSIVE PERIODONTITIS}

Generalized aggressive periodontitis usually affects individuals under age 30, but older patients also may be affected. Clinically, GAP is characterized by generalized interproximal attachment loss affecting at least three permanent teeth other than first molars and incisors. The radiographic picture in GAP can range from severe bone loss associated with the minimal number of teeth, as described previously, to advanced bone loss affecting the majority of teeth in the dentition. ${ }^{13}$

A striking feature of AP is the lack of clinical inflammation despite the presence of deep periodontal pockets and advanced bone loss. Furthermore, in many cases, the amount of plaque on the affected teeth is minimal, which seems inconsistent with the amount of periodontal destruction present. ${ }^{13}$

\section{EXCLUSION CRITERIA}

- Uncontrolled systemic diseases

- Pregnant and lactating mothers

- Overhanging restorations

- Interproximal caries

- Orthodontic appliances

- Occlusal disharmony at the area of bone loss.

\section{EXAMINER STANDARDIZATION}

Two calibrated examiners (SS, NS), who were well trained prior to the study, performed all the examinations, and the examiners followed a written protocol that described the measurement methods and the sequence of assessments. The inter-examiner reliability was assessed in a subsample of 10 subjects, who were selected randomly within the study group. The subjects were reexamined within a few hours after the first examination, and replicate measurements of probing depth and attachment loss were evaluated. The reproducibility of periodontal measurements was assessed. The measurement errors of probing depth and attachment loss were estimated by calculating the standard deviation of the differences between replicate measurements for the individual site assessments.

\section{CLINICAL EXAMINATION}

A total number of 500 subjects were screened to estimate the prevalence of AP in patients aged 13 to 60 years (mean age $36.5 \pm 33.2$ years), who visited the OPD of Periodontology, RajaRajeswari Dental College \& Hospital, Bengaluru, Karnataka, India. Thorough gingival examination, including bleeding on probing, was done to evaluate the gingival condition. The periodontal status was evaluated by assessment of the clinical attachment level, probing pocket depth, and gingival recession. The subjects provisionally diagnosed with AP were referred to the Department of Radiology for further examination. The radiographic assessment involved full mouth intraoral periapical radiographs and panoramic radiographs.

\section{OTHER RELEVANT CLINICAL FINDINGS}

Apart from the diagnostic criteria for AP, the following clinical findings were also recorded:

- Mobility

- Pathologic migration

- Missing tooth.

\section{RADIOGRAPHIC FINDINGS}

- Arc-shaped loss of the alveolar bone extending from the distal surface of the second premolar to the mesial surface of the second molar presenting as a mirror image pattern in oral pantomogram (Fig. 1).

- Wider bone defects.

\section{STATISTICAL ANALYSIS}

Prevalence of AP in patients was established by tabulating the results in an Excel sheet and dividing the final

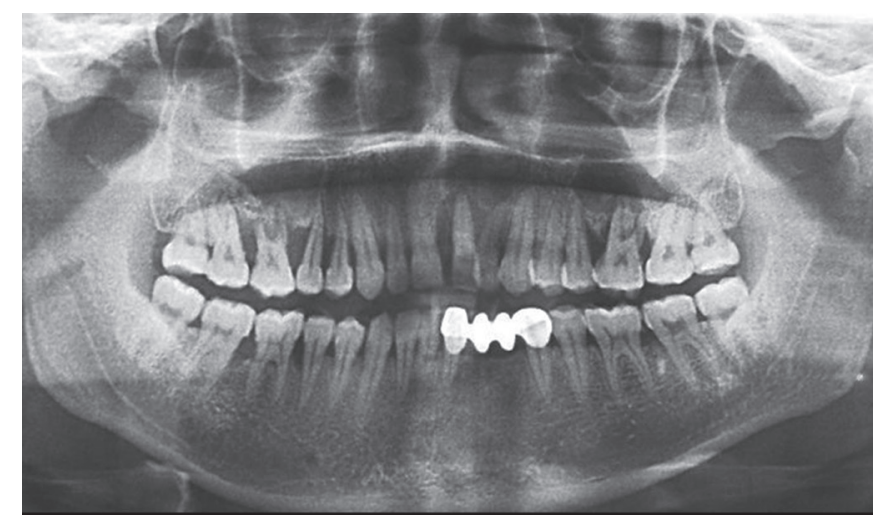

Fig. 1: Oral pantomogram showing pattern of bone destruction in AP 
Table 1: Prevalence of localized and generalized forms of AP with gender distribution

\begin{tabular}{lll}
\hline Gender & Male & Female \\
\hline LAP & 2 & 2 \\
GAP & 3 & 1 \\
\hline Total & 5 & 3 \\
\hline
\end{tabular}

confirmed cases by the number of subjects screened and expressed as percentage.

\section{RESULTS}

\section{Prevalence of AP}

A total number of 500 systemically healthy subjects were screened in the field, and the distribution of various forms of periodontal diseases gender wise is depicted in Table 1. A total number of eight cases were finally confirmed to have either LAP or GAP (four cases in each respectively), giving a prevalence percentage of $1.6 \%$. Among the eight cases of $\mathrm{AP}$, three were females and five were males.

\section{DISCUSSION}

This epidemiological cross-sectional study was undertaken to determine the prevalence of AP in patients visiting the OPD of Periodontology, RajaRajeswari Dental College \& Hospital, Bengaluru, Karnataka, India. This study consisted of 500 patients, including 220 males and 280 females. Eight out of 500 patients were diagnosed with AP in either localized or generalized form. The generalized form was more common than the localized form.

In the present study, attachment loss was considered as the key parameter for the diagnosis of cases along with definite radiographic evidence of bone loss. In addition to a threshold of $>4 \mathrm{~mm}$ for attachment loss, measurements made at six sites per tooth were considered as diagnostic criteria for categorization of AP.

The present study showed a prevalence of $1.6 \% \mathrm{AP}$ and is in accordance with many studies by Rao and Tewani, ${ }_{14}^{14}$ India 6.80\%; Albandar et $\mathrm{al}_{1}^{15} 28.8 \%$; Priscila et al ${ }_{1}^{16}$ Brazil 9.9\%; and Imran and Ataa, ${ }_{17}^{17}$ Yemen $2.6 \%$. On the contrary, various authors have reported a very modest prevalence of AP as follows: Kronauer et al, ${ }^{18}$ Switzerland $0.9 \%$; Saxby, ${ }^{19}$ West Midlands $1 \%$; and Van der Velden et $\mathrm{al}^{20}{ }^{20}$ Amsterdam $0.1 \%$.

The wide variations in global prevalence from 0.1 to $28.8 \%$ only indicate the different methodological strategies and stress the imperative need for more strict criteria before a disease can be ascribed to the category of AP. When prevalence was assessed based on the socioeconomic status, some researchers reported higher prevalence of LAP in low socioeconomic groups (Gjermo et $\mathrm{al}^{21}{ }^{21} 3.8 \%$; Lopez et $\mathrm{al}^{22} 0.32 \%$; Susin and
Albandar, $\left.{ }^{23} 5.5 \%\right)$, but another study showed no significant difference between socioeconomic groups. ${ }^{15}$ In the present study, most of the subjects with AP belonged to poor to moderate economic groups. However, as the study analyzed only eight cases, perceptible conclusions could not be drawn about the relationship between the socioeconomic status and the prevalence of disease.

The higher prevalence of AP in the present study can be attributed to the fact that the population studied is hospital-based because of the convenience (convenient sample). The prevalence assessed among these types of samples will be higher than that assessed among the general population as persons with dental problems attend hospitals, and they are not representative of the general population. The prevalence in the hospital-based population is about $10 \%$ higher as compared with that in the general population.

The report in the present study indicates that a higher percentage of males are affected with AP than females. But since only a total of 500 patients were analyzed, a conclusion cannot be drawn between the gender affected and AP.

\section{LIMITATIONS OF THE STUDY}

The present study considered only a total of 500 subjects. Future studies with screening involving more number of subjects would be more reliable and predictive of the prevalence percent of AP.

\section{CONCLUSION}

The prevalence rate of AP in the screened population is $1.6 \%$. This value suggests a higher prevalence but is consistent with most global prevalence rates. The prevalence of AP globally is widely variable, ranging from 0.1 to $28.8 \%$, indicating diverse approaches to clinical examination and criteria employed and racial, geographical, socioeconomic, and possibly genetic susceptibility factors.

\section{REFERENCES}

1. Armitage GC. Development of a classification system for periodontal diseases and conditions. Ann Periodontol 1999 Dec;4(1):1.

2. Black, GV. Diseases of the peridental membrane having their beginning at the margin of the gum. In: Litch, WF., editor. American System of Dentistry. Vol. 1. 2nd ed. Philadelphia: Lea Brothers; 1886. p. 953-979.

3. Demmer RT, Papapanou PN. Epidemiologic patterns of chronic and aggressive periodontitis. Periodontol 20002010 Jun;53:28-44.

4. Nassar MM, Afifi O, Deprez RD. The prevalence of localized juvenile periodontitis in Saudi subjects. J Periodontol 1994 Jul;65(7):698-701.

5. Hansen BF, Gjermo P, Beigwitz-Larsen KR. Periodontal bone loss in 15 year old Norwegians. J Clin Periodontol 1984 Feb;11(2):125-131. 
6. GjermoP, Bellini HT, Pereira Santos V, Martins JG, FerracyoliJR. Prevalence of bone loss in a group of Brazilian teenagers assessed on bite-wing radiographs. J Clin Periodontol 1984 Feb;11(2):104-113.

7. Albandar JM. Prevalence of incipient radiographic periodontal lesions in relation to ethnic background and dental care provisions in young adults. J Clin Periodontol 1989 Nov;16(10):625-629.

8. Timmerman MF, Van der Weijden GA, Armand S, Abbas F, Winkel EG, Van Winkelhoff AJ, Van Der Velden U. Untreated periodontal disease in Indonesian adolescents, clinical and microbiological baseline data. J Clin Periodontol 1998 Mar;25(3):215-224.

9. Albandar JM, Brown LJ, Loe H. Clinical features of early onset periodontitis. J Am Dent Assoc 1997 Oct;128(10): 1393-1399.

10. Devi K. Prevalence of juvenile among school children in Hyderabad - An epidemiological study [dissertation]. Hyderabad: Osmania University; 1984. p. 38-39.

11. Suresh K. Epidemiological investigation into the status of oral hygiene and periodontal disease in tribal and urban population of Andhra Pradesh [dissertation]. Vijayawada:Dr. NTR University of Health Sciences; 1995. p. 34-35.

12. Reddy SR. Prevalence of localized aggressive periodontitis in Khammam urban population [dissertation]. Vijayawada:Dr. NTR University of Health Sciences; 2010. p. 37-38.

13. Armitage GC. Development of a classification system for periodontal diseases and conditions. Ann Periodontol 1999 Dec;4(1):1.

14. Rao SS, Tewani SV. Prevalence of periodontosis among Indians. J Periodontol 1968 Jan;39(1):27-34.
15. Albandar JM, Muranga MB, Rams TE. Prevalence of aggressive periodontitis in school attendees in Uganda. J Clin Periodontol 2002 Sep;29(9):823-831.

16. Corraini P, Pannuti CM, Pustiglioni AN, Romito GA, Pustiglioni FE. Risk indicators for aggressive periodontitis in an untreated isolated young population from Brazil. Braz Oral Res 2009 Apr-Jun;23(2):209-215.

17. Imran AG, Ataa MAS. Prevalence of aggressive periodontitis among Yemeni students from schools in the city of Thamar. Rev Sul-Bras Odontol 2010 Jul-Sep;7(3):325-331.

18. Kronauer E, Borsa G, Lang NP. Prevalence of incipient juvenile periodontitis at age 16 years in Switzerland. J Clin Periodontol 1986 Feb;13(2):103-108.

19. Saxby MS. Juvenile periodontitis: An epidemiological study in the west Midlands of the United Kingdom. J Clin Periodontol 1987 Nov;14(10):594-598.

20. Van der Velden U, Abbas F, Van Steenbergen, De Zoete OJ, Hesse M, De Ruyter C, De LaatVH, De Graaff J. Prevalence of periodontal breakdown in adolescents and presence of Actinobacillus actinomycetemcomitans in subjects with attachment loss. J Periodontol 1989 Nov;60(11):604-610.

21. GjermoP, Bellini HT, Pereira Santos V, Martins JG, FerracyoliJR. Prevalence of bone loss in a group of Brazilian teenagers assessed on bite-wing radiographs. J Clin Periodontol 1984 Feb;11(2):104-113.

22. Lopez R, Fernandez O, Jara G, Baelum V. Epidemiology of clinical attachment loss in adolescents. J Periodontol 2001 Dec;72(12):1666-1674.

23. Susin C, Albandar JM. Aggressive periodontitis in an urban population in southern Brazil. J Periodontol 2005 Mar;76(3):468-475. 